\title{
Lumbar L4-L5 ganglion cyst with hemicauda equina syndrome. Report of a case and review of the literature
}

\author{
Gabriel Iacob $^{1}$, Bogdan Rotund ${ }^{2}$ \\ ${ }^{1}$ Prof. PhD, Neurosurgery Clinic, UMPh “Carol Davila” Bucharest, University \\ Hospital Bucharest, Romania \\ ${ }^{2}$ Senior neurosurgeon, University Hospital Bucharest, Romania
}

\begin{abstract}
Mostly associated with degenerative facet joints and spondylolisthesis, symptomatic lumbar juxta facet cysts ganglion and synovial cysts are uncommon lesions of the spine. They can mimic herniated discs, causing low back pain, radiculopathy or even cauda equina syndrome; also some of them are associated with spinal instability. Referring to a case with hemicauda equina syndrome where microsurgical decompression was performed with very good result; alternative treatment solutions are discussed: conservative therapy for symptomatic lesions is often unsuccessful, spinal fusion should be made for those cases with spinal instability, although there are reports of spontaneous resolution of these cysts as well as the symptoms associated with them.
\end{abstract}

Key words: ganglion cyst, synovial cyst, juxtafacet cyst, degenerative spine disease, cauda equine syndrome, spinal fusion.

\section{Introduction}

Although juxtafacet cysts are lesions noted as incidental findings associated with spinal facet joints on imaging studies of the spine (computed tomography - CT and magnetic resonance imaging - MRI); in some patients, they may produce symptoms: nerve root and/or spinal cord compression (1-9). In the lumbar area juxta facet cysts are mostly associated with degenerative facet joints and spondilolisthesis, generating back and radicular pain. Patients with extradural degenerative lesions associated with with cauda equina syndrome generated by a juxtafacet cyst are rare (10). Despite histological differences between ganglion and synovial cysts, physicians refer to both entities as synovial cysts because there are no differences concerning treatment and prognosis (11-14).

\section{Method}

A female aged 47 years old with a hemicauda equina syndrome, with intense left radiculopathy: left motor L5, S1 (-4) deficits and sensory disturbances, no bowel and bladder dysfunction, installed four days before admission. There was no history of trauma. Before surgery the patient had received: simple and flexion X-P: normal, without associated spinal instability; lumbar spinal computed tomography: round, left, low density cyst at L4-L5, in contact with the left articular process, compressing L5 root. Magnetic resonance imaging (figure 1) in T2 weighted sequences shows a left, epidural, hyperintense mass at L4-L5, compressing root and dura, in contact with 
the left articular process and the bulging disc L4. On sagital T2 weighted sequences, the outer wall appear hypointense. The patient was operated using a left hemilaminectomy suited by a microsurgical resection of the lumbar cyst, sparing the facet joint, without surgery related complications. Lateral left recess was adequately decompressed as the L5 and S1 nerve roots. It was one cyst, without attachment to the left articular process. The removed cyst were histologically evaluated. Macroscopic findings (figure 2 A) has shown a spherical, 1.3 diameter cyst, slate gray-pink, renitent consistence, well delineated wall, containing on section a fibrous capsule limiting yellow, thick, jellylike, mucinous fluid. The microscopic findings (figure $2 \mathrm{~B}, \mathrm{C}$ ) has shown a wall cyst of fibrous connective tissue, a fluid with high concentration of albumin, globulin and hyaluronic acid, without hemorrhagic material or hemosiderin deposits; no synovial lining membrane was found.

\section{Results}

Patient postoperative period was uneventful; one week after, no pain, without neurologic deficits. 2 months after surgery, she has no pain, no instability and on a new MRI, there was no case of a recurrent cyst.

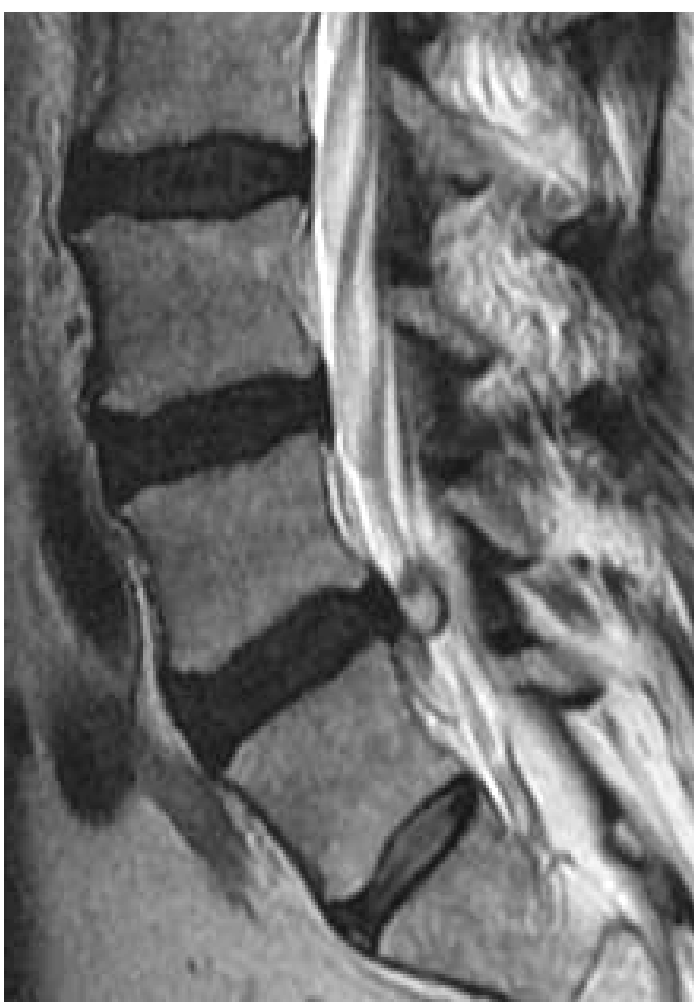

A

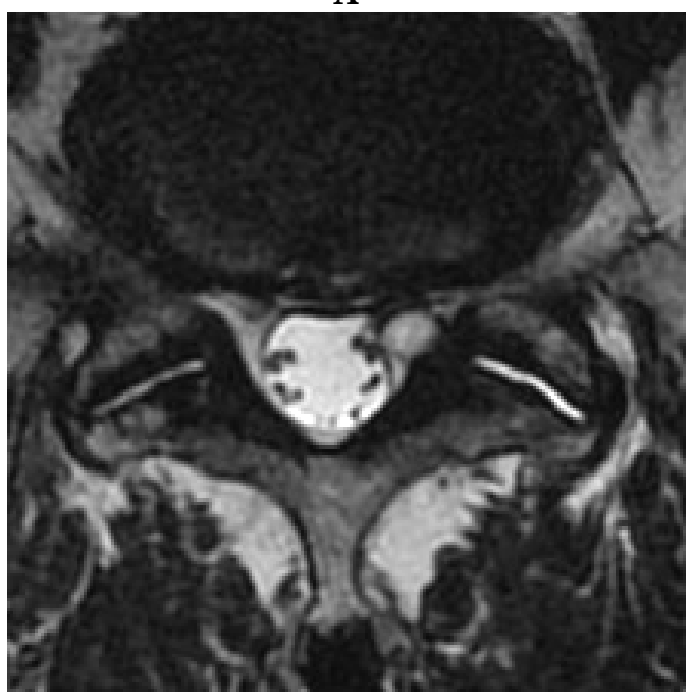

B

Figure 1

A. Lumbar spinal MRI in T2 weighted sequences shows a left, epidural, hyperintense mass at L4-L5, compressing root and dura, in contact with the left articular process and the bulging disc L4. B. Sagital

T2 weighted sequences, the outer wall appear hypointense. 


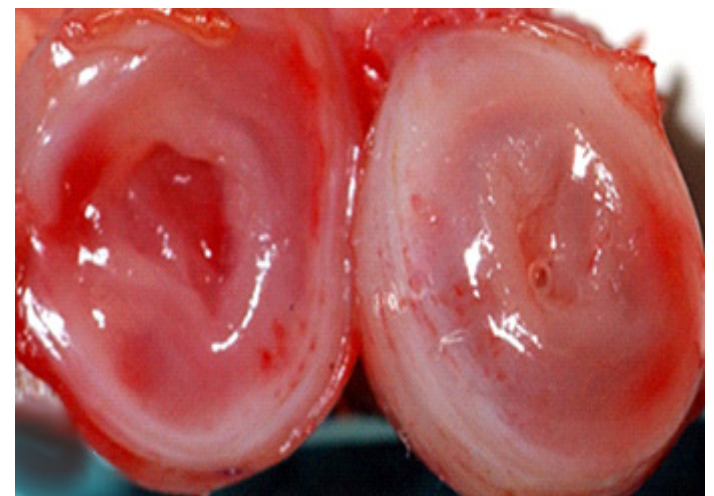

A

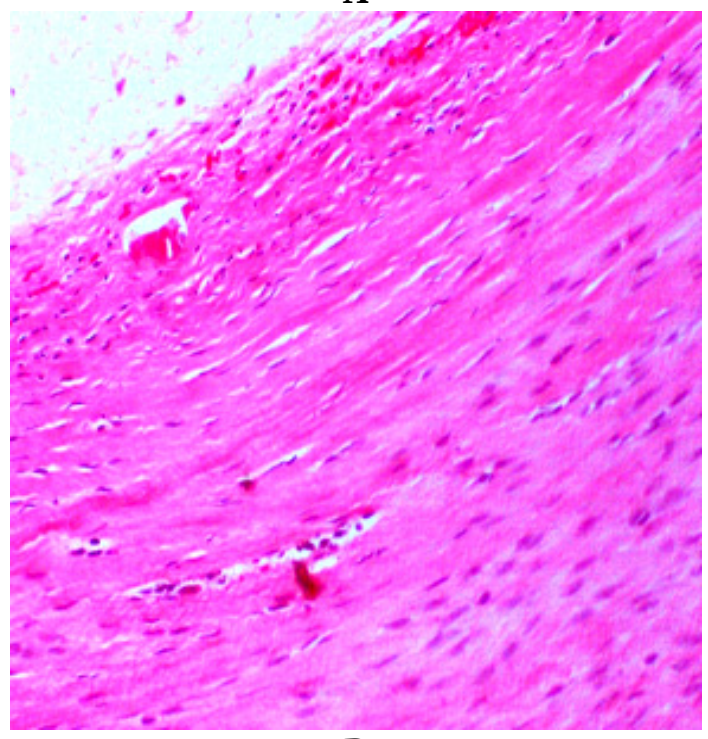

B

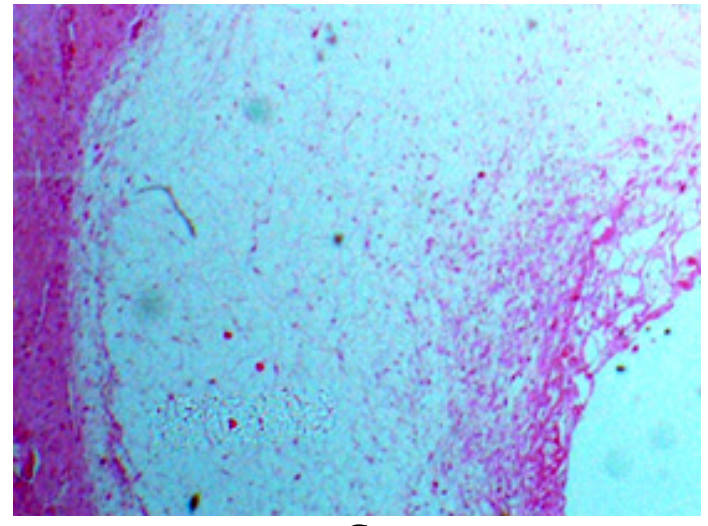

C

Figure 2

Resected cyst, macroscopic and microscopic findings: A. macroscopic view of the cyst, B,C ganglion cyst wall (pseudocyst): absence of synovial cell lining with connective tissue and foci of myxoid degeneration

\section{Discussion}

Based on Nabors classification for spinal meningeal cysts (15) there a three main groups:

- meningeal cysts: epidural without neural tissue, epidural with neural tissue similar terms perineural or Tarlov cysts, intradural (anterior, posterior or lateral of the thoracal spinal cord, leated to a trauma, a hemorrhage or inflammation with secondary arachnoiditis, communicants or non communicants)

- epidural, non meningeal cysts include juxta facet cysts (synovial and ganglionar cysts), traumatic pseudomeningocele intra or extraspinal, containing or not roots, also tumoral lesions with cysts: schwannomas, dermoids, pigmented villonodular synovitis, metastasis

- neuroenteric cysts: rare, intradural, anterior extramedullary, uni or polilobular, interesting several spinal segments, frequently associated with congenital spinal anterior vertebral anomalies also KlippelFeil syndrome, diastematomyelia, spina bifida.

Ayberk von Gruker in 1880 (cited by 6) in a postmortem examination, reported the first description of a spinal synovial cyst and Baker 1885 (cited by 9) explain synovial cysts adjacent to articulations. Vosschulte and Börger (16) first described in 1950 spinal synovial cysts as entities found arising at or around the joint capsule of the spinal facets, that cause symptoms by compressing nerves and many years the terms: synovial cyst, ganglion cyst, pseudocysts were used synonymously. Kao (17)(18) describe in 1968 and in 1974 proposed the term juxta-facet (juxta $=$ near or nearby) cysts; including the entities of synovial and ganglion cysts to the spinal facet joints or arising from the ligamentum 
flavum. Distinction between these two types of cysts could be difficult, also clinically unimportant (9) (19) (20). Hsu (21) reffer to intra-spinal facet cysts regardless of whether synovial lining cells present or not, but with similar clinical manifestation. Although there are histological differences between synovial and ganglion cysts, their treatment and prognosis are identical. That is why they are grouped together as juxtafacet cysts or in the stricter sense of word "cystic formations of mobile spine" (=CYFMOS), but in practice it is common for physicians to refer to both entities as synovial cysts (22). Disposed near articulations, either attached or adjacent to spinal facet joints $(1,3-5,7$, $11,12,21-23)$, spinal synovial or ganglion cysts are relatively small lesions - mean diameter $1 \mathrm{~cm}$, found incidentally in all spinal segments: most frequently in the lumbar spine (88-99\%), especially at the most mobile interspace L4-L5 (12), up to $8 \%$ arise in the thoracic region and 1 to $4 \%$ are commonly found in the cervical area (3$5,24)$. Although juxtafacet cysts are usually unilateral, they are found bilaterally, most commonly at the L4-L5 level, in up to 54\% of patients in some series, associated with significant spinal instability and the potential need for spinal fusion. More precisely these cysts could be placed: ventromedial to the facets as in our case, dorsal to the facet joints (and often asymptomatic), in the interlaminar space, with facet attachment - most common, without nerve root compression; dorsal midline uncommon, with involvement of dura \& the base of neural arch; at the interspinous ligament, clinically unimportant or attached to inner side of ligamentum flavum, without facet attachment. Their occurrence and location seem to be associated with degenerative arthritis of facet joints in up to $90 \%$ and with spondylolisthesis in up to $100 \%$ of patients in some series $(1,2,6-9$, 21).

On anatomo - pathological grounds juxta-facet cysts are:

- synovial cysts represent a true cyst. They are small cystic growths that occur adjacent to and attached to synovial tissuelined joints, such as the spinal facet joints with a narrow connecting pedicle, which connects their cystic centers to the cavities of their parent joints, lined by a true synovium, contain synovial fluid. They are not only found in the spine, but also in the knees, hips, and elbows. Grossly, synovial cysts contain clear, serous or xanthochromic fluid and occasionally have calcifications within their walls. Histologically, these lesions have a normal synovial lining, but they may also contain hemosiderin, giant cells or inflammatory cells. Synovial cysts may also contain hemosiderin deposits (occasional hemmorhage) because they occasionally bleed within themselves, causing acute growth and exacerbation of symptoms; or even calcification $(1-8,22)$

- ganglion cysts are pseudocysts, occur in moveable tissues (the most common site of ganglion cyst is wrist, also knees, feet and elbows), are similar in size to synovial cysts, but lack a true synovial lining, more spherical, have no or little attachment to joint; as a result of advanced degeneration of soft spinal tissue or from a synovial cyst after degeneration or destruction of synovial lining membrane due to a chronic trauma. Grossly, ganglion cysts contain viscous, proteinaceous fluid. On histological examination, their walls are composed of well-vascularized fibrous connective tissue. Cyst fluid is thicker and 
more viscous than normal synovial fluid; it could be highly viscous, sticky, jelly-like mucinous fluid or clear; never contain hemorrhagic materials $(21,22)$.

The etiology of JFC is unknown $(1,6$, $12)$; several possibilities may play a role in the pathogenesis:

- synovial fluid extrusion through a defect or rupture in the joint capsule - most likely theory, even by latent growth of a developmental rest. Increased motion and chronic micro trauma are favorable factors

- myxoid degeneration and cyst formation in the collagenous connective tissue - various sites

- fibroblasts proliferation with increased hyaluronic acid production and secondary cyst formation

- pluripotential, non specific mesenchymal cell proliferation

Depending on topography, juxtafacet cysts are crowding the spinal canal, compressing the spinal cord and causing myelopathy, neurogenic claudication. In the lumbar area, in consequence of a lumbar spine stress (exercise loading) in addition to a degenerative lesion of the soft lumbar tissue synovial and ganglion cyst are uncommon cause of dorso-lateral root compression, associated or not with lateral recess and spinal stenosis $(6,23)$. Symptoms depend on the pattern of growth, especially at L4-L5: upward and anterior growth, compresses L4 root, downward extension and intraspinal expansion compresses L5 root, extraspinal, dorsal to the facet joints growth could be asymptomatic - majority. Patients with juxtafacet cysts present insidiously or acutely, due to intracystic or epidural hemorrhage from the lesion $(10,25,26)$ with one or more of the following conditions: lower back pain with dull ache in the back radiating to the hip, unilateral or bilateral radiculopathy, neurogenic claudication, lumbar extension is usually restricted but lumbar flexion is maintained, motor deficit (12\%), sensory loss (26\%) reflex changes (12), cauda equina syndrome. Rarely hemorrhage occur into the cyst, mostly in the lumbar region with acute intractable radiculopathy that is the result of sudden increase in the cyst's volume with hematoma. Bleeding in the cyst is the result of spontaneous rupture of fragile neoangiogenic vessels of the cyst's wall, although their rupture in traumatic events and anticoagulant therapy has so been reported causing an acute cauda equina syndrome (10).

Juxtafacet cysts were usually discovered incidentally during surgery before the advent of CT and MR imaging, but with the increasing use of neuroimaging in the evaluation and management of cases of degenerative disease of the spine, they are commonly seen on preoperative diagnostic studies. Their presence and potential role as a generator of symptoms must be considered (1-4, 6, 7, 11, 12, 21-23). Juxtafacet cysts were associated with disc herniations, other epidural mass, degenerative spinal instability as well as other elements of degenerative spine disease. Diagnostic studies should include: dynamic flexion/extension X-ray, computed tomography (CT) and magnetic resonance imaging (MRI). CT scans reveal juxtafacet cysts as a soft tissue low-density, round lesions adjacent to facets, occasionally with calcification within their outer walls at the articulation level, projecting into the epidural space postero-lateral after contrast we can see contours. On CT myelography, contrast material is typically not seen within the center of a juxtafacet cyst. 
MRI is the tool of choice for diagnosis of juxtafacet cysts (4), with a typical pattern consisting of a hyperintense center and hypointense rim, adjacent to the facet joints on T2-weighted sequences. Their proteinaceous contents may appear hyperintense to cerebrospinal fluid. On T1weighted MR imaging sequences, juxtafacet cysts appear as hypointense masses, most commonly located dorsal to the neural elements in the lumbar spinal canal. In contrast, herniated discs are commonly located ventral to the neural elements. Their outer walls may enhance weakly on both CT and MR imaging studies $(12,14)$. In the MRI myelography the lesion appeared as a round extradural posterolateral filling defect. In case of bleeding cysts there are different aspect of MRI imaging; ex: for subacute hemorrhage in the cyst we can saw inhomogeneous/isointense centers, intracystic hemorrhage can be predicted radiologically by an MRI (inhomogeneous center in T2-weighted images and irregular hyperintensity in T1-weighted images) (10, $23,24)$, but there is no correlation with the acute onset of symptoms. Because of similar aspect for synovial or ganglionar cysts on MRI, it's mandatory to precise that only histology may differentiate them (4, 21, 22).

Optimal treatment for juxta facet cysts is a matter of controversy $(1,6,7,11,12,19$, $21,27,28,30,34,35)$. Although relevant reports in the international literature are increasing, the controversy about conservative versus surgical treatment and the need for concomitant fusion still exists. The optimal treatment is not known. There are several attitudes:

- Conservative treatment has been proposed by certain authors, including: bed rest, symptomatic treatment: analgesic medications, spine stabilization exercises, lower-extremity stretching, orthopedic corsets with disappointing results (27). Therefore there are observations that some of these cysts may decrease in size or collapse spontaneously, similar to symptomatic disc herniations, which can regress over time (28). There is four cases reports of cysts that resolved spontaneously $(29,30)$, but it is infrequent. Another conservative procedures for management of intraspinal juxtafacet cysts are: percutaneous CT-guided puncture cyst aspiration plus intracystic injection of steroid drugs (31) - associated with frequent recurrence, transforaminal epidural injections of steroid agents may be effective in temporary relieving the inflammatory component; however, enlarging a mass lesion with in the spinal canal may also be associated with risks of neural compromise. There is little information available on the efficacy and outcome with treatment of lumbar facet joint synovial cysts by percutaneous, fluoroscopic, facet joint steroid injections, with cyst distention and rupture with shortterm improvement or no improvement at all (32). Several complications such as dural puncture, spinal nerve injury, bleeding, and infection have been reported (33).

- Surgical resection is the treatment of choice for symptomatic juxtafacet cysts in all cases of intractable pain or neurologic deficit (1, 3, 5-9, 11-13, 19, 20, 34-37). Under general anesthesia, in the prone position, a wide exposure is encouraged to achieve complete resection hemilaminectomy on the symptomatic side is performed, suited by partial medial facetectomy, excision of thickened ligamentum flavum, foraminotomy. There 
are series of patients with juxta facet cysts treated with minimally invasive decompression in conjunction with hemilaminotomy and cyst excision through a serial dilator/tubular surgical retractor system $(6,36,37)$. Because their usual location is in the lateral recess, microscopic piecemeal excision of the compressing cyst involves decompression of the traversing nerve root at that level as well as the exiting root above, along with adequate de compression of the lateral recess without fusion $(13,36,37)$. Sometimes removal can be difficult, because juxtafacet cysts are often firmly adherent to the duramater; some authors recommend careful application of electrocautery to the facet joint to prevent recurrence (12). Partial cyst resection may lead to recurrent lesions and to a return of symptoms $(12,20,38,39)$. For those cases with degenerative listhesis, with associated instability primary fusion after resection of these lesions should be considered (1, 3, 6-8, 11-13, 19, 21, 22, 3539). Similar to a herniated disc, lumbar stenosis, or spondylolisthesis, these lesions are not the cause, but instead a manifestation of spinal instability that may or may not be clinically significant (34). Many authors reported that no difference in surgical outcome was found between patients having fusion and those who did not have it, while others concluded that, a concomitant fusion procedure may be performed in selected cases $(19,20,38,39)$. Métellus et al. (12) has concluded that there is no reliable criterion that allows the development of a symptomatic spinal instability to be predicted in patients with preoperative spondylolisthesis and therefore, fusion as a first line procedure is still debatable. Others have mentioned an association between spinal cysts and spondylolisthesis/instability and better surgical outcomes in patients having fusion than in those who did not have it $(20,21)$. Closure is done with a submuscular drain). Using these technique, generally all patients got benefit $(70 \%-80 \%$ good results up to $100 \%)(38)$, with few related complications (dural tears in up to $4 \%$ of primary surgeries and up to $12 \%$ of secondary surgical procedures), with very good surgical outcomes and short duration of hospitalization (three days) (39).

\section{Conclusions}

Juxtafacet cysts (ganglion or synovial) are uncommon lesions of the spine with unclear etiology: micro trauma, a manifestation of spinal instability, more frecquent in the lumbar area, especially at L4-L5 level, generating lumbar radicular pain or even cauda equine syndrome. MRI is the neuroinvestigation tool of choice for diagnosis. Medical management (even puncture of the cyst) is less successful than it is for a herniated nucleus pulposus, does not adequately improve symptoms in patients with intraspinal juxta facet cysts and radicular signs.

Microsurgical resection of the cyst is the best treatment strategy, a safe and effective treatment for those symptomatic intraspinal juxta facet cysts unresponding to conservative treatment, associated with excellent long-term outcome also very low morbidity. For surgical exposure and complete decompression of the neural elements, as little bone removal as is deemed necessary to allow safe dissection of adhesions to the duramater is sufficient. Symptomatic patients due to mechanical instability before surgery or in cases where wide bone decompression was required, a fusion should be considered to solve 
iatrogenic instability, never as a first line procedure. Recurrences and surgical complications are rare.

\section{References}

1.Lyons M.K., Atkinson J.L. et al. - Surgical evaluation and management of lumbar synovial cysts: the Mayo Clinic experience, J. Neurosurg. 2000, 93(1 Suppl): 537

2.Tillich M., Trummer M., et al. - Symptomatic intraspinal synovial cysts of the lumbar spine: Correlation of MR and surgical findings, Neuroradiology 2001, 43:1070-5

3.Trummer M., Flaschka G., et al. - Diagnosis and surgical management of intraspinal synovial cysts: report of 19 cases, J Neurol Neurosurg Psychiatry 2001, 70:74-77

4.Schmid G., Willburger R., et al. - Lumbar intraspinal juxtafacet cysts. MR imaging and CT-arthrography, Rofo 2002, 174:1247-52

5.Nakamura S., Miyauchi H., et al. - Spinal Juxta-Facet Cyst : Report of Seven Cases and Review of the Literature, Clinical Orthopaedic Surgery 2004, 39, 1, 85-92

6.Epstein N. - Lumbar synovial cysts: a review of diagnosis, surgical management and outcome assessment, J Spinal Disord Tech 2004, 17:321-325

7.Choudhri H.F., Perling L.H. - Diagnosis and management of juxtafacet cysts, Neurosurg Focus 2006, 20 (3):E1, 1-4

8.Ayberk G., Özveren F., et al. - Lumbar synovial cysts: Experience with nine cases, Neurol Med Chir (Tokyo) 2008, 48:298-303

9.Lommers E., Gillet P. - Le cas clinique du mois: une cause inhabituelle de sciatalgie necessitant un traitement chirurgical: le kyste arthrosynovial lombaire, Rev. Med. Liege, 2010, 65, 12: 665-668.

10.Abolfazl R. - Bleeding in a Lumbar Juxtafacet Cyst with Cauda Equina Syndrome: Report of a Case and Review of the Literature, WScJ 2011, 2: 46-51

11.Boviatsis E.J., Stavrinou L.C., et al. - Spinal synovial cysts: Pathogenesis, diagnosis and surgical treatment in a series of seven cases and literature review. Eur Spine J. 2008, 17:831-7

12.Métellus P., Fuentes S., et al. - Retrospective study of 77 patients harbouring lumbar synovial cysts: functional and neurological outcome, Acta Neurochir (Wien) 2006, 148(1):47-54

13.Deinsberger R., Kinn E., Ungersböck K. Microsurgical treatment of juxta facet cysts of the lumbar spine, J Spinal Disord Tech. 2006, 19(3):155-60 14.Christophis P., Asamoto S., et al. - "Juxtafacet cysts", a misleading name for cystic formations of mobile spine
(CYFMOS), Eur Spine J. 2007, 16(9): 1499-1505

15.Nabors MW, Pait TG, et al. - Updated assessment and current classification of spinal meningeal cysts., J Neurosurg. 1988, Mar, 68(3):366-77

16.Vosschulte K., Borger G. - Anatomische und funktionelle untersuchungen uber den bandscheibenprolaps, Langenbecks Arch Chir 1950, 265: 329-355

17.Kao C.C., Uihlein A., Bickelr W.H.- Lumbar Intraspinal Extradural Ganglion Cyst, J. Neurosurg. 1968, 29: 168-72

18.Kao C.C.,Winkler S.S., Turner J.H. - Synovial Cyst of Spinal Facet, J.Neurosurg 1974, 41: 372-6

19.Pirotte B., Gabrovsky N., Massager N., et al. Synovial cysts of the lumbar spine: Surgery-related results and outcome. J Neurosurg. 2003, 99(1 Suppl):14-9

20.Bydon A., Xu R., et al. - Recurrent back and leg pain and cyst reformation after surgical resection of spinal synovial cysts: Systematic review of reported postoperative outcomes, Spine J. 2010, 10:820-6

21.Hsu K.Y., Zucherman J.F. et al. - Lumbar intraspinal synovial and ganglion cysts (facet cysts). Ten-year experience in evaluation and treatment. Spine 1995, 20:80-9

22.Christophis P., Asamoto S., et al. - "Juxtafacet cysts", a misleading name for cystic formations of mobile spine (CYFMOS), Eur Spine J. 2007, 16(9): 1499-1505

23.Doyle A.J., Merrilees M. - Synovial cysts of the lumbar facet joints in a symptomatic population: Prevalence on magnetic resonance imaging, Spine 2004, 29:874-8

24.Miwa M., Doita M., et al: - An expanding cervical synovial cyst causing acute cervical radiculopathy, J Spinal Disord Tech 2004, 17:331-333

25.Wait S.D., Jones F.D. et al. - Symptomatic epidural hematoma caused by lumbar synovial cyst rupture: report of two cases and review of the literature, Neurosurgery 2005, 56: E1157

26.Wang Y.Y., McKelvie P, et al. - Trauma as a precipitant of haemorrhage in synovial cysts. J Clin. Neurosci. 2004, 11:436-439

27.Shah R.V., Lutz G.E. - Lumbar intraspinal synovial cysts: Conservative management and review of the world's literature, Spine J. 2003, 3:479-88

28.de Beukelaar J.W., Dallenga A., et al. - Juxta facet cyst of the lumbar spine: an uncommon cause of lumbar radiculopathy, Ned Tijdschr Geneeskd. 2010, 154:A1673

29.Swartz P.G., Murtagh F.R. - Spontaneous resolution of an intraspinal synovial cyst. AJNR 2003, 24:12611263

30.Houten J.K., Sanderson S.P., et al.: Spontaneous regression of symptomatic lumbar synovial cysts. Report of three cases, J. Neurosurg 2003, 99 (2 
DOI: 10.2478/romneu-2013-0017

Suppl):235-238

31.Lim A.K., Higgins S.J., et al. - Symptomatic lumbar synovial cyst: Management with direct CT-guided puncture and steroid injection, ClinRadiol. 2001, 56:990-3

32.Allen T.L, Tatli Y, Lutz G.E. - Fluoroscopic percutaneous lumbar zygapophyseal joint cyst rupture: A clinical outcome study, Spine J. 2009, 9:387-95

33.Martha J.F., Swaim B., et al. - Outcome of percutaneous rupture of lumbar synovial cysts: A case series of 101 patients, Spine J. 2009, 9:899-904

34.Epstein N. - Lumbar laminectomy for the resection of synovial cysts and coexisting lumbar spinal stenosis or degenerative spondylolisthesis: an outcome study, Spine 2004, 29:1049-1056

35.El Shazly A.A., Khattab F.M. - Surgical excision of a
Juxtafacet cyst in the lumbar spine: A report of thirteen cases with long-term follow up, Asian J. Neurosurg. 2011, 6(2): 78-82

36.Sandhu F.A., Santiago P., et al: - Minimally invasive surgical treatment of lumbar synovial cysts. Neurosurgery 2004, 54:107-112

37.Oertel M.F., Ryang Y. et al. - Microsurgical therapy of symptomatic lumbar juxta facet cysts. Minim Invasive Neurosurg. 2003, 46(6): 349-353.

38.Banning C.S., Thorell W.E., et al. - Patient outcome after resection of lumbar juxtafacet cysts, Spine 2001, 26:969-972

39.Khan A.M, Synnot K., et al. - Lumbar synovial cysts of the spine: An evaluation of surgical outcome, J Spinal Disord Tech. 2005, 18:127-31 